\title{
Role of ubenimex as an anticancer drug and its synergistic effect with Akt inhibitor in human A375 and A2058 cells
}

This article was published in the following Dove Press journal:

OncoTargets and Therapy

\section{Xiaoqing Wang' \\ Yang Liu' \\ Rongde $\mathrm{Wu}^{\prime}$ \\ Feng Guo' \\ Lijuan Zhang' \\ Mingyu Cui' \\ Xiangyu $\mathrm{Wu}^{\prime}$ \\ Yongfei Zhang ${ }^{2}$ \\ Wei Liu'}

'Department of Pediatric Surgery, Shandong Provincial Hospital affiliated to Shandong University, Jinan, People's Republic of China; ${ }^{2}$ Department of Dermatology, Shandong Provincial Qianfoshan Hospital affiliated to Shandong University, Jinan, People's Republic of China
Correspondence: Wei Liu

Department of Pediatric Surgery, Shandong Provincial Hospital affiliated to Shandong University, 324 Jingwu Street, Jinan, Shandong 25002I,

People's Republic of China

$\mathrm{Tel}+86$ I5 I 6886389 I

Email lemontreeIII9@I26.com

Yongfei Zhang

Department of Dermatology, Shandong Provincial Qianfoshan Hospital affiliated to Shandong University, Jingshi Load, 16766 Jinan, Shandong, People's Republic of China

Tel +86 I358905 0847

Email13589050847@I63.com
Background: Malignant melanoma (MM) is a malignant tumor produced by changes in melanocytes in the skin or other organs. In the classification of skin tumor mortality, skin melanoma ranks the highest. Ubenimex, an Aminopeptidase N (APN) inhibitor, is now widely used for cancer as an adjunct therapy, conferring antitumor effects. Apoptosis and the induction of autophagy have both been found to be closely associated with tumor cell death.

Methods: In this study, the A375 and A2058 cell lines were treated with ubenimex. Cell viability was measured using the Cell Counting Kit 8 assay. Apoptosis and autophagic cell death were assessed using flow cytometry and acridine orange/ethidium bromide staining. Protein expression was assessed by Western blot analyses and immunofluorescence. Matrigel invasion and migration assays were used to examine the metastatic ability of melanoma cells.

Results: The results revealed that ubenimex inhibited the expression of APN in melanoma cells, which may be connected with the inhibition of metastasis. In addition, it increased melanoma cell death by inducing apoptosis and autophagic cell death. This effect was accompanied by increased levels of $\mathrm{p}-\mathrm{JNK}$. Moreover, treatment with ubenimex induced protective Akt activation, and combined use of an Akt inhibitor with ubenimex provided a better effect for inducing tumor cell death.

Conclusion: As an effective anti-tumor drug in vitro, ubenimex might be an excellent adjunctive therapy for the treatment of melanoma, with greater effects when combined with the use of an Akt inhibitor.

Keywords: melanoma, ubenimex, jnk, Akt, mixed cell death, metastasis

\section{Introduction}

Malignant melanoma (MM) is a malignant tumor produced by changes in melanocytes in the skin or other organs. In the classification of skin tumor mortality, skin melanoma ranks the highest. ${ }^{1}$ Skin melanoma manifests a significant change in pigmented lesions within months or years. In recent years, the incidence of malignant melanoma has posed a huge threat to human health. ${ }^{2}$ The disease is characterized by a high rate of metastasis in the early stage, poor sensitivity to chemotherapy, and poor prognosis. ${ }^{3}$ Therefore, understanding the reasons for chemotherapy treatment resistance and exploring possible adjuvant medications is the final "life-saving straw" for melanoma patients, especially those with advanced cases.

Ubenimex, also known as bestatin, has been used as an adjunct therapy for many tumors, enhancing the function of immunocompetent cells and conferring antitumor effects, ${ }^{4}$ and the effect is also Aminopeptidase N (APN) related. APN, also called $\mathrm{CD} 13$, is involved in various cellular processes, and, in particular, it has been 
revealed to correlate with the invasion/metastasis of various malignancies. ${ }^{4}$ In malignant melanoma, the inhibition of APN always induces the inhibition of metastasis. ${ }^{5}$ However, few studies have examined the functions of ubenimex in melanoma cells in vitro.

Cell death is divided into programmed cell death and nonprogrammed death. Programmed cell death, which is often called apoptosis, is caspase-dependent cell death, whereas autophagic cell death is caspase independent. ${ }^{6}$ In many cases, autophagy is unequivocally the mode of tumor cell death. ${ }^{7}$ The JNK pathway plays an important role as a classical signaling pathway in the regulation of tumor cell apoptosis and autophagic death. ${ }^{8}$ The function of JNK as a regulator in apoptosis and autophagic cell death has been demonstrated in many tumor cells, such as bladder tumor, osteosarcoma, breast cancer, and hepatocellular carcinoma. ${ }^{8-11}$ In melanoma, JNK also plays a vital role in proliferation and cell death. ${ }^{12}$ The Akt pathway is often regulated in response to DNAdamaging chemotherapeutics, and participates in regulating tumor cell death. ${ }^{13}$ One of our previous papers also revealed its functions in tumor cell death and radiotherapy resistance following treatment with ubenimex. ${ }^{14}$

Referring to the drug ubenimex, our previous study proved its efficacy in renal cell carcinoma, prostate cancer, and glioma cells, ${ }^{14-16}$ although all experiments indicated that ubenimex could work as an anti-cancer drug, the mechanisms differ. Therefore, this study aimed to investigate whether ubenimex could still work as an anti-tumor drug in malignant melanoma cells and to determine the underlying potential mechanisms.

\section{Materials and methods}

\section{Tumor cell lines}

Malignant melanoma cell lines A375 and A2058 were purchased from the Chinese Academy of Sciences (Beijing, People's Republic of China) Cell Bank. Cells were maintained in Dulbecco's Modified Eagle's Medium (DMEM), a high-glucose medium (Macgene, Beijing, People's Republic of China), supplemented with $1 \%$ penicillin-streptomycin and $10 \%$ fetal bovine serum (Biological Industries, Kibbutz Beit Haemek, Israel). The cells were incubated at $37^{\circ} \mathrm{C}$ in a humidified atmosphere with $5 \% \mathrm{CO}_{2}$.

\section{CCK-8 cell proliferation assay}

A375 and A2058 cells in an exponential phase of growth were harvested and seeded into 96-well plates at a density of 6,000 cells/well in DMEM high-glucose medium supplemented with different concentrations of ubenimex. After the cells underwent specific treatments for the indicated time periods, $10 \mu \mathrm{L}$ of Cell Counting Kit-8 (CCK-8) solution (CCK-8 cell proliferation and cytotoxicity assay kit-8; Dojindo, Kumamoto, Japan) was added to each well. The plates were then incubated for an additional $2 \mathrm{~h}$ at $37^{\circ} \mathrm{C}$, and the absorbance was determined using a microplate reader (EL340; Bio-Tek Instruments, MA, USA) at $450 \mathrm{~nm}$.

\section{Acridine orange/ethidium bromide double staining}

Cells were cultured in six-well plates for $24 \mathrm{~h}$ and then treated with different doses of ubenimex for $48 \mathrm{~h}$. After the indicated treatment times, the acridine orange/ethidium (AO/EB) (Leagene, Beijing, China) operating fluid was mixed with reagents $\mathrm{A}$ ( $\mathrm{AO}$ solution), $\mathrm{B}$ (EB solution), and $\mathrm{C}$ ( $\mathrm{AO} / \mathrm{EB}$ Dilution Buffer) at a ratio of 1:1:8. To each sample, $2 \mu \mathrm{L}$ of $\mathrm{AO} / \mathrm{EB}$ operating fluid was added, and the supernatant was discarded after low-speed centrifugation at $500 \mathrm{~g} / \mathrm{min}$. The cells were then resuspended in $\mathrm{AO} / \mathrm{EB}$ dilution buffer at a concentration of $5 \times 10^{6}$ cells $/ \mathrm{mL}$, and $1 \mu \mathrm{L}$ of $\mathrm{AO} / \mathrm{EB}$ operating fluid was added to $25 \mu \mathrm{L}$ of cell suspension. Next, the cells were observed under a fluorescence microscope (Nikon Inc., Tokyo, Japan).

\section{Western blot analysis}

To determine APN, LC3B, JNK/P-JNK, Akt/P-Akt, P62, and cleaved-caspase-3/pro-caspase-3 levels, proteins were extracted from the cells by suspension in radioimmunoprecipitation assay buffer. Samples were centrifuged at $12,000 \mathrm{rpm}$ at $4^{\circ} \mathrm{C}$ for $30 \mathrm{~min}$, and the supernatants were recovered for analysis. The protein concentrations were determined using the Bradford protein method and the bicinchoninic acid protein assay kit (Sigma, St Louis, MO, USA). Protein $(40 \mu \mathrm{g})$ was electrophoresed on a pre-cast bis-Tris polyacrylamide gel $(8 \% \sim 12 \%)$ and then transferred onto a polyvinylidene difluoride membrane. Membranes were blotted with rabbit anti-APN $(1: 1,000)$, rabbit antiLC3B (1:500), rabbit anti-JNK $(1: 1,000)$, rabbit anti-pJNK $(1: 1,000)$, rabbit anti-Akt $(1: 1,000)$, rabbit anti-p-Akt $(1: 1,000)$, mouse anti-P62 $(1: 1,000)$, rabbit anti-Caspase-3 $(1: 1,000)$ (all from Abcam, San Francisco, CA, USA), and mouse anti-actin (1:1,000; Proteintech, NY, USA), followed by horseradish peroxidase-conjugated secondary antibodies (1:5,000; ZsBio, Beijing, China). Immunoblots were visualized using enhanced chemiluminescence (LAS-4000).

\section{Annexin V-phycoerythrin (PE)/ 7-aminoactinomycin D staining}

The apoptotic cells were quantified (\%) using an Annexin $\mathrm{V}$-PE/7-aminoactinomycin D (7AAD) kit (BD Biosciences, 
NJ, USA) and detected by flow cytometry. The A375 and A2058 cells were harvested after $48 \mathrm{~h}$ of treatment with ubenimex or combined with Akt inhibitor. Next, the cells were resuspended in a binding buffer $(10 \mathrm{mmol} / \mathrm{L}$ 4-(2-hydroxyethyl)-1-pipera-zine-ethanesulfonic acid, $140 \mathrm{mmol} / \mathrm{L} \mathrm{NaCl}$, and $2.5 \mathrm{mmol} / \mathrm{L} \mathrm{CaCl}_{2}, \mathrm{pH} 7.4$ ) and incubated with Annexin V-PE/7AAD in the dark for $15 \mathrm{~min}$. A total of 10,000 cells/sample were analyzed using a FACSCalibur or an EPICS XL flow cytometer (BD Biosciences).

\section{Immunofluorescence}

We performed cell concentration smears and fixed the cells with $4 \%$ paraformaldehyde for $20 \mathrm{~min}$. We thoroughly rinsed the cells with $0.01 \mathrm{M} \mathrm{PBS}(5 \min \times 3)$ and incubated the cells in $0.3 \%$ Triton at room temperature for $20 \mathrm{~min}$, followed by a wash in $0.01 \mathrm{M}$ PBS (5 min $\times 3$ ). Next, $30 \mu \mathrm{L} /$ sample goat serum blocking solution was added at room temperature for $60 \mathrm{~min}$, followed by a 1:100 dilution of primary antibody at $30 \mu \mathrm{L} /$ sample (diluted in goat serum). The cells were placed in a wet box at $4^{\circ} \mathrm{C}$ overnight and then washed in $0.01 \mathrm{M}$ PBS $(5 \min \times 3)$. On the next day, $4^{\prime}, 6$-diamidino2-phenylindole was added for $10 \mathrm{~min}$, and the cells were thoroughly rinsed with $0.01 \mathrm{M}$ PBS $(5 \mathrm{~min} \times 3)$, followed by the addition of 1:100 fluorescent secondary antibody at $30 \mu \mathrm{L} /$ sample ( $10 \%$ skim milk powder) at room temperature and in the dark for $60 \mathrm{~min}$. Next, the cells were washed in $0.01 \mathrm{M}$ PBS $(5 \min \times 3)$ and mounted with an anti-fluorescence quencher.

\section{Wound healing migration assays}

The A375 and A2058 cells were plated in six-well culture plates and grown to $100 \%$ confluency before scratching with a sterile P200 pipette tip across the monolayer. The cell debris was removed by washing with PBS, and the cells were cultured in DMEM and 2\% fetal bovine serum (FBS) supplemented with different concentrations of ubenimex. The area of the scratch was measured at different times (A375 for 0 h, 12 h, 24 h; A2058 for 0 h, 18 h, $36 \mathrm{~h}$ ), and quantification was performed by measuring the area of cell migration at different time points compared to the scratch area at $0 \mathrm{~h}$. Each experiment was repeated three times.

\section{Matrigel invasion assay and matrigel migration assay}

Migration assays were performed using transwell chambers. Control untreated cells or cells treated with ubenimex ( 0.5 or $1 \mathrm{mg} / \mathrm{mL}$ for $48 \mathrm{~h}$ ) were trypsinized, and $1 \times 10^{5}$ cells were plated in the upper wells in serum-free medium, while medium with $10 \%$ FBS was added to the lower well as a stimulus. After $24 \mathrm{~h}$ of incubation, the cells on the matrigel side of the chambers were removed using a cotton swab. The inserts were fixed in methanol and stained using hematoxylin and eosin. The number of invading cells attached to the other side of the inserts was quantified under a light microscope using six random fields at a magnification of $\times 200$. In addition, invasion assays were performed using transwell chambers that were pre-coated with $40 \mu \mathrm{L}$ of $1 \mathrm{mg} / \mathrm{mL}$ matrigel matrix (BD Biosciences, Bedford, MA, USA). Each treatment group had a control containing $2.5 \times 10^{5}$ cells plated in the upper wells, with the remaining steps following the matrigel migration assay.

\section{Statistical analysis}

The data were statistically analyzed using Student's $t$-test, the $\chi^{2}$ test, or Fisher's exact test using SPSS version 19.0 software (IBM Corporation, Armonk, NY, USA). A $P$-value $<0.05$ was considered significant.

\section{Results \\ Ubenimex could induce cell death and reduce the vitality of melanoma cells}

CCK-8 was used to examine cell proliferation after treatment with different doses of ubenimex for different time periods in A375 and A2058 cells (Figure 1). The proliferation of tumor cells decreased over time. In addition, the proliferation of melanoma cells was significantly inhibited by the high dose of ubenimex after $48 \mathrm{~h}$ of exposure. Using CCK-8, we determined the appropriate concentration and time of drug use. Then, cell death was examined by $\mathrm{AO} / \mathrm{EB}$ staining and flow cytometry (Figures 2 and 3). The cell death rate increased with ubenimex treatment, which showed a dose-dependent response (Figure 3). Therefore, we concluded that ubenimex could inhibit cell proliferation and induce cell death in both A375 and A2058 cells.

\section{Through transwell invasion and migration assays, we concluded that ubenimex could reduce invasion mobility in A375 cells}

Wound healing assay and transwell assays were performed to determine whether ubenimex could affect the migration and invasion capacity of A375 cells (Figures 4 and 5). The migration capacity of A375 cells was significantly suppressed by ubenimex in a concentration-dependent manner after $48 \mathrm{~h}$ of exposure. Moreover, the dose of ubenimex used was also an important factor affecting A375 cells. We then further examined the effect of ubenimex on the 

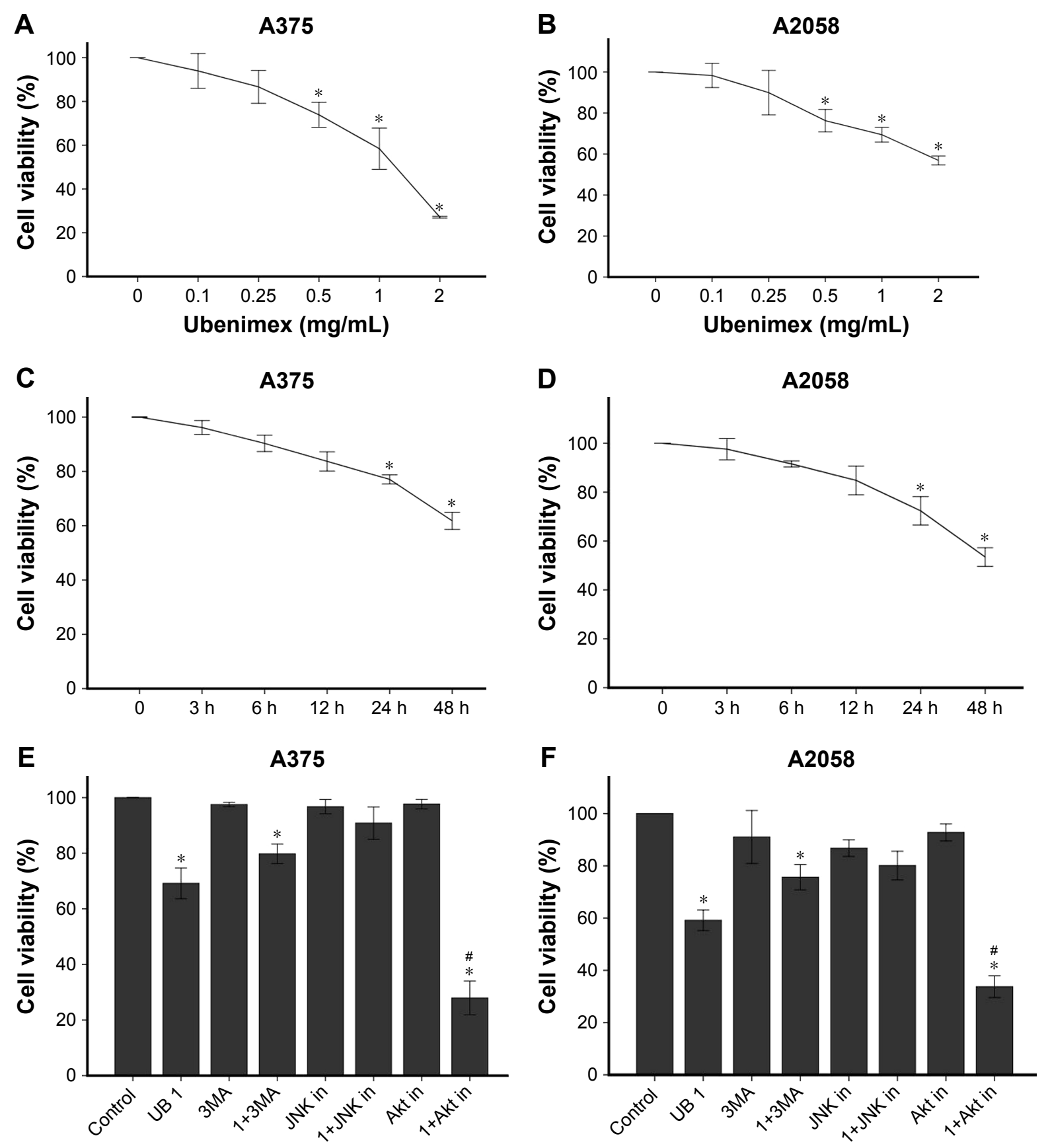

Figure I Ubenimex inhibits the cell proliferation of A375 and A2058 cells.

Notes: $(\mathbf{A}$ and B) CCK8 proliferation assay was performed after a $48 \mathrm{~h}$ culture of A375 or A2058 cells with the indicated concentrations of ubenimex. $* P<0.05$ for untreated control cells compared to all ubenimex doses. (C and D) The CCK8 proliferation assay of A375 or A2058 cells was performed with a I mg/mL dose of ubenimex at different times. ${ }^{*} \mathrm{P}<0.05$ for untreated control cells compared to all time points. (E and $\mathbf{F}$ ) The CCK8 proliferation assay of A375 or A2058 cells was performed with different treatments (control; ubenimex I mg/mL for $48 \mathrm{~h}$; $3 \mathrm{MA}$ for $48 \mathrm{~h}$; ubenimex I mg/mL and 3MA for $48 \mathrm{~h}$; JNK inhibitor for $6 \mathrm{~h}$; ubenimex I mg/mL and JNK inhibitor for $6 \mathrm{~h}$, and then ubenimex I mg/mL for $42 \mathrm{~h}$; Akt inhibitor $48 \mathrm{~h}$; Akt inhibitor and ubenimex I mg/mL for $48 \mathrm{~h}$ ). *P<0.05 for statistically significant vs untreated control cells. ${ }^{P}<0.05$ for statistically significant combined effect of Akt inhibitor and ubenimex I mg/mL for $48 \mathrm{~h}$ vs ubenimex I mg/mL alone. Data are expressed as the mean \pm SD of three independent experiments.

Abbreviations: CCK8, Cell Counting Kit-8; UB, ubenimex; JNK, c-Jun N-terminal kinase; 3MA, 6-Amino-3-methylpurine.

invasive activity of A375 cells using matrigel invasion assays.

Pretreatment with ubenimex markedly inhibited the invasive capacity of the A375 cells. Taken together, these results suggest that ubenimex inhibited the migration and invasion of A375 cells.

\section{Ubenimex could function as an APN inhibitor in A375 and A2058 cells}

Western blotting was used to test the expression of APN after ubenimex treatment (Figure 6). Additionally, A375 and A2058 cells were treated with ubenimex $(1 \mathrm{mg} / \mathrm{mL})$ for 
A
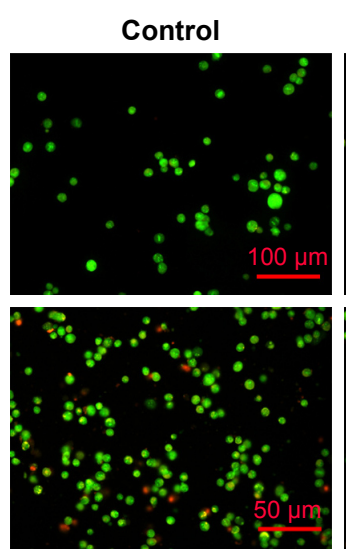

B

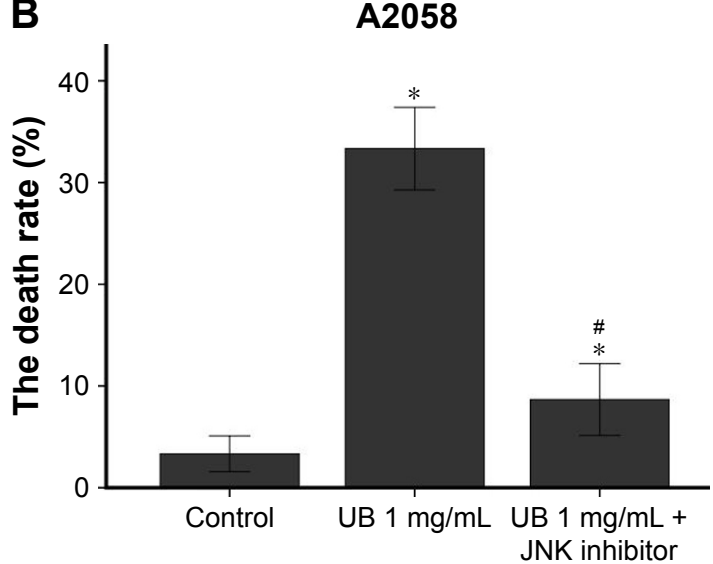

UB $1 \mathrm{mg} / \mathrm{mL}+$ JNK inhibitor
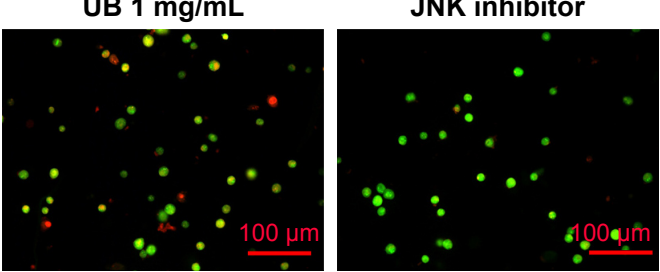

A2058
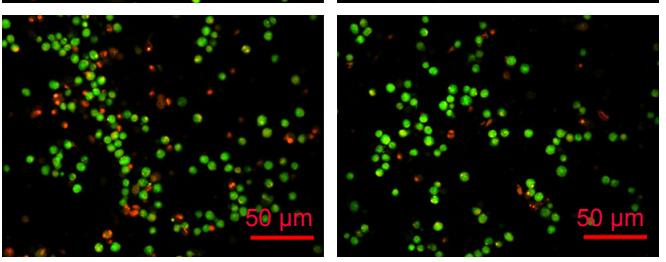
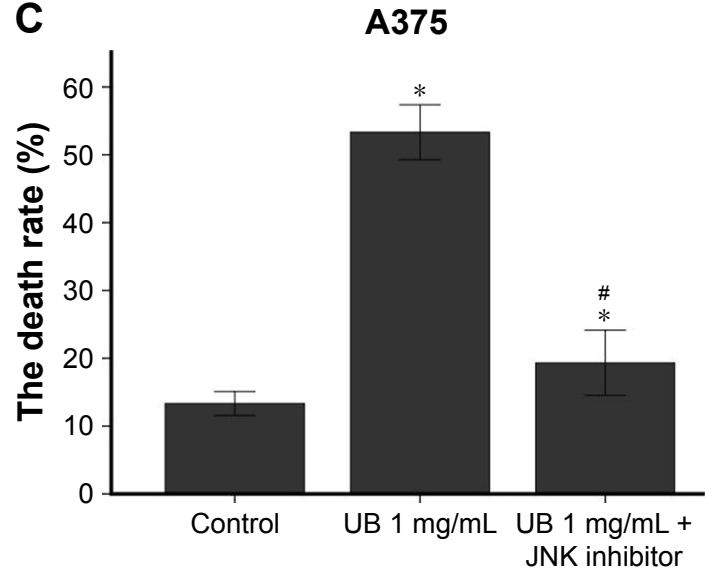

Figure 2 AO/EB staining shows the cell death in both A375 and A2058 cells.

Notes: (A) A375 and A2058 were treated with ubenimex $(\mathrm{I} \mathrm{mg} / \mathrm{mL})$ combined with/without JNK inhibitor. The cells of the third group were treated with ubenimex $(\mathrm{I} \mathrm{mg} / \mathrm{mL})$ and JNK inhibitor for $6 \mathrm{~h}$, and then ubenimex $(\mathrm{I} \mathrm{mg} / \mathrm{mL})$ alone for $42 \mathrm{~h}$. After $48 \mathrm{~h}$, fluorescence microscopy was used to examine the death rate of each group. The death rates of A375 (B) and A2058 (C) cells per one field of a high-power microscope as determined by acridine orange staining are shown. $* P<0.05$ vs the control and $\# P<0.05$ vs only with ubenimex $(\mathrm{I} \mathrm{mg} / \mathrm{mL})$. Data are expressed as the mean $\pm \mathrm{SD}$ of three independent experiments.

Abbreviations: $A O / E B$, acridine orange/ethidium bromide; $U B$, ubenimex.

$48 \mathrm{~h}$. The expression of APN was significantly inhibited by the increased dose of ubenimex.

\section{Treatment with ubenimex induced apoptosis in melanoma cells}

Flow cytometry is a well-known experimental method for detecting cell apoptosis. As shown in Figure 3, the death rates of A375 and A2058 cells increased after ubenimex treatment and proved to be dose dependent. In addition, AO/EB staining also showed increased death rate of cells after treatment with ubenimex (Figure 2). In addition to autophagic cell death, apoptosis increased after the treatment. The CCK- 8 assay provided additional proof: when we added 3MA to inhibit autophagy, the cell death induced by ubenimex persisted. Programmed cell death, which is often called apoptosis, is caspase-dependent cell death. Therefore, to further examine the increase in apoptosis, Western blotting was used to examine the level of caspase-3 (Figure 6). Treatment with ubenimex increased the level of cleaved-caspase-3, which is also evidence of increased cell apoptosis.

\section{The cell death induced by ubenimex included not only apoptosis, but also autophagic cell death}

The cell death induced by ubenimex is a combination of effects. In addition to apoptosis, autophagic cell death also occurs in melanoma cells. As mentioned above, 3MA was used to distinguish apoptosis from autophagic cell death. After the combined use of 3MA and ubenimex, the death rate was significantly decreased (Figure 1). We can easily conclude that ubenimex induced autophagic cell death in melanoma cells. Additionally, we used Western blotting and immunofluorescence to detect the expression of LC3B, an iconic protein in autophagy (Figures 6 and 7). As the dose of ubenimex increased, the expression of LC3B and autophagyrelated P62 also increased. 
A
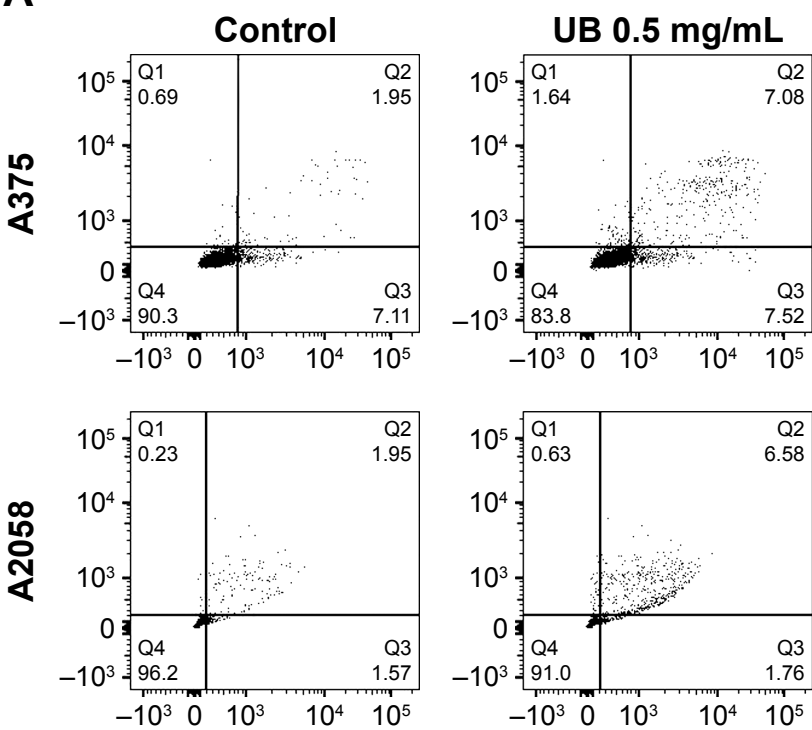

B

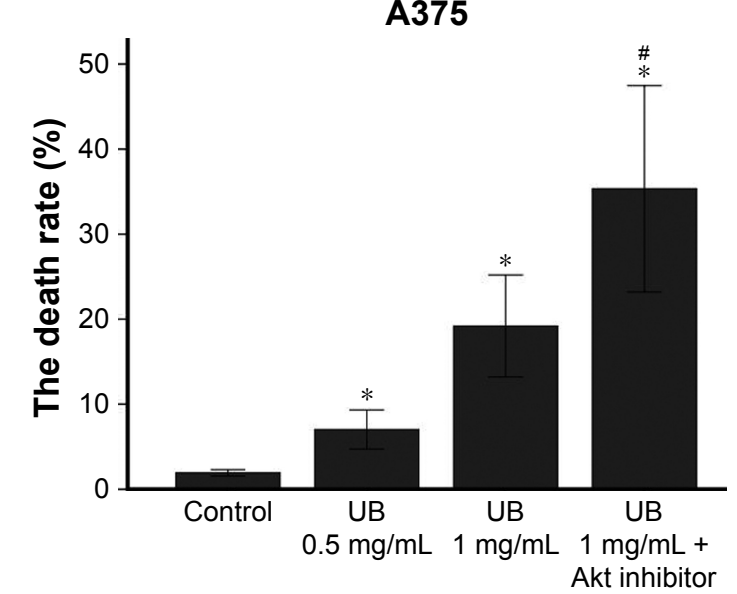

UB $1 \mathrm{mg} / \mathrm{mL}+$

Akt inhibitor
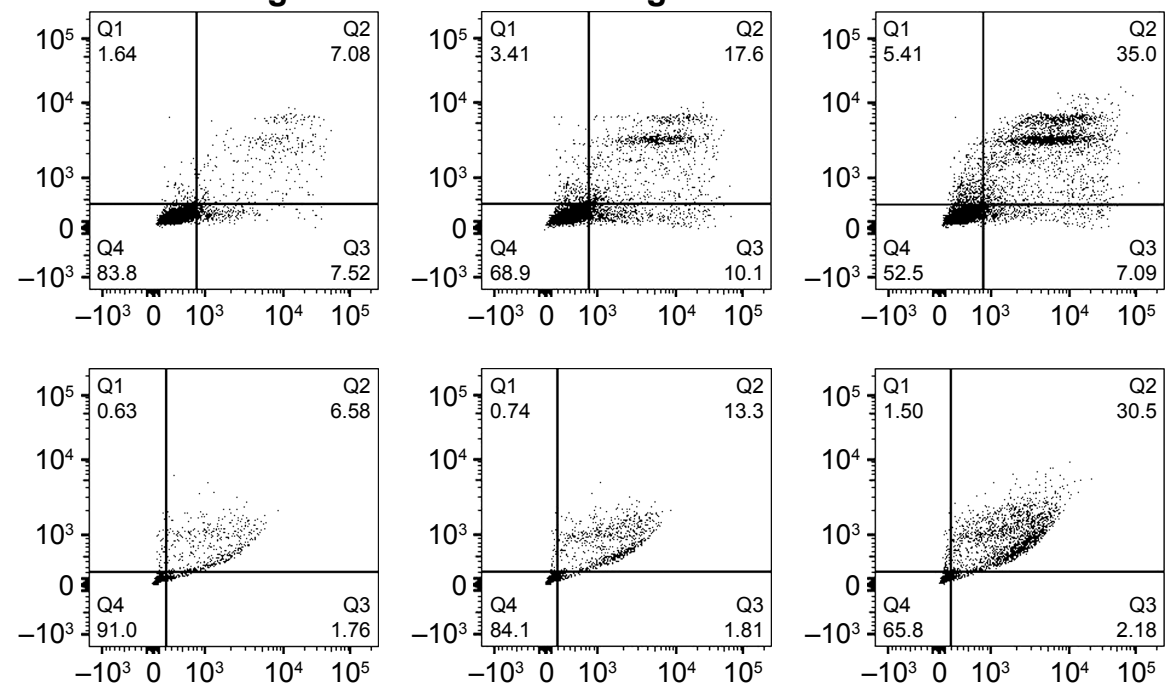

C

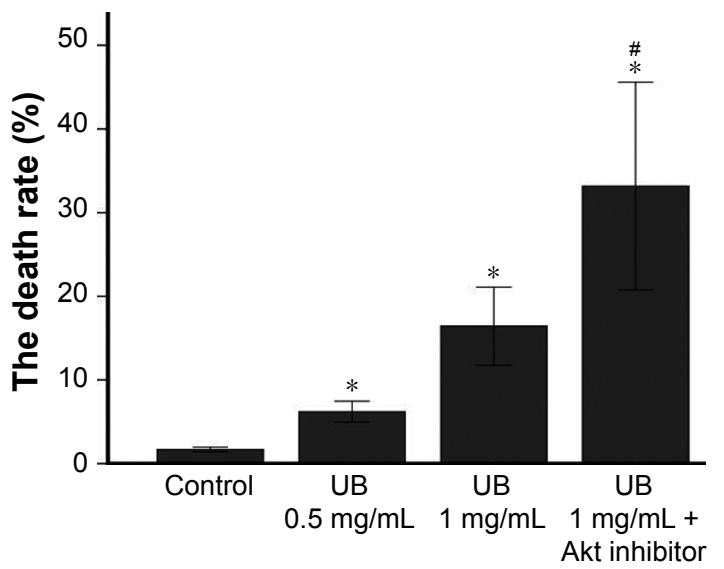

Figure 3 Ubenimex induces cell death in both A375 and A2058 cells.

Notes: (A) A375 and A2058 cells were treated with ubenimex $(0.5 \mathrm{mg} / \mathrm{mL}$ and I mg/mL) and combined use of ubenimex (I mg/mL) and Akt inhibitor for $48 \mathrm{~h}$, and the death rate was then detected using flow cytometry. Cells that are in late apoptosis or already dead are both Annexin V-PE and 7-AAD positive (Q2), (B and C) Autophagic cell death and apopotosis-related cell death (both $A 375$ and $A 2058$ ), showed by $Q 2$ were measured together by flow cytometry. $* P<0.05$ vs control and $\# P<0.05$ vs only with ubenimex $(\mathrm{I} \mathrm{mg} / \mathrm{mL})$. Data are presented as the mean $\pm \mathrm{SD}$ of three independent experiments.

Abbreviation: UB, ubenimex.

\section{The induced cell death may be regulated by the variation of $\mathrm{p}-\mathrm{JNK}$ expression}

We used Western blotting to examine the level of p-JNK (Figure 8). After treatment with ubenimex $(1 \mathrm{mg} / \mathrm{mL})$ for different lengths of time ( $0,3 \mathrm{~h}, 6 \mathrm{~h}, 12 \mathrm{~h}, 24 \mathrm{~h}, 48 \mathrm{~h})$, we examined the variation in p-JNK1 and p-JNK2. Despite slight differences, the expressions of these two proteins both increased after treatment with ubenimex at early time points and then decreased to a level lower than at $0 \mathrm{~h}$. To further illustrate this point, we chose the 6-h time point for Western blot verification (Figure 9). We obtained the same result: the expression of p-JNK1 and p-JNK2 increased following treatment with ubenimex for $6 \mathrm{~h}$. Moreover, CCK-8 was used to examine the role of the JNK pathway in melanoma cell death (Figure 1). We chose to use a JNK inhibitor (SP600125, Selleck, Houston, TX, USA) in combination with ubenimex for $6 \mathrm{~h}$, and then used ubenimex alone for $42 \mathrm{~h}$. The results showed decreased cell death in A375 and A2058 cells.

\section{Treatment with ubenimex could}

\section{induce the Akt pathway, which shows}

\section{a protective effect for melanoma cells}

After treatment with different doses of ubenimex for $48 \mathrm{~h}, \mathrm{p}$-Akt was significantly increased in a dose-dependent manner (Figure 6). Then, we detected the expression at different time points $(0,3 \mathrm{~h}, 6 \mathrm{~h}, 12 \mathrm{~h}, 24 \mathrm{~h}, 48 \mathrm{~h})$ after treatment with ubenimex (1 mg/mL) (Figure 8$)$. p-Akt was also significantly increased. Then, we added an Akt inhibitor 
A

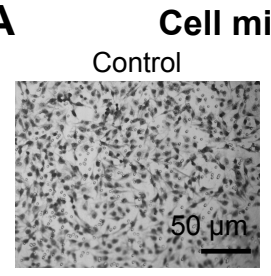

Cell migration assay of $\mathrm{A} 375$

UB $0.5 \mathrm{mg} / \mathrm{mL}$
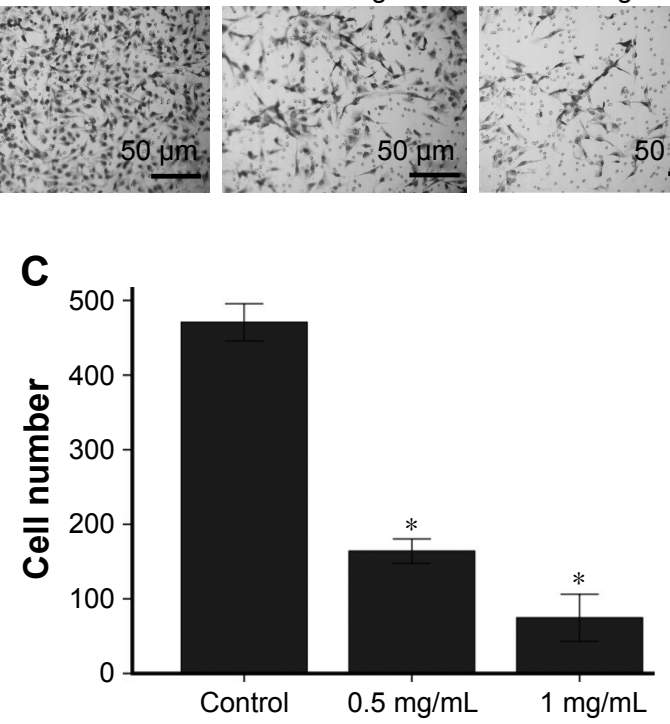

UB $1 \mathrm{mg} / \mathrm{mL}$

B Cell invasion assay of A375

Control

UB $0.5 \mathrm{mg} / \mathrm{mL}$

UB $1 \mathrm{mg} / \mathrm{mL}$

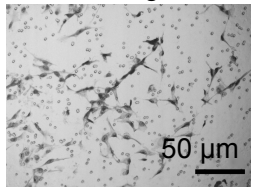

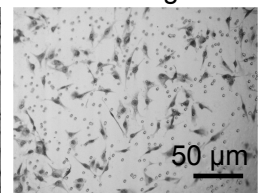

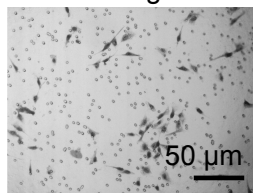

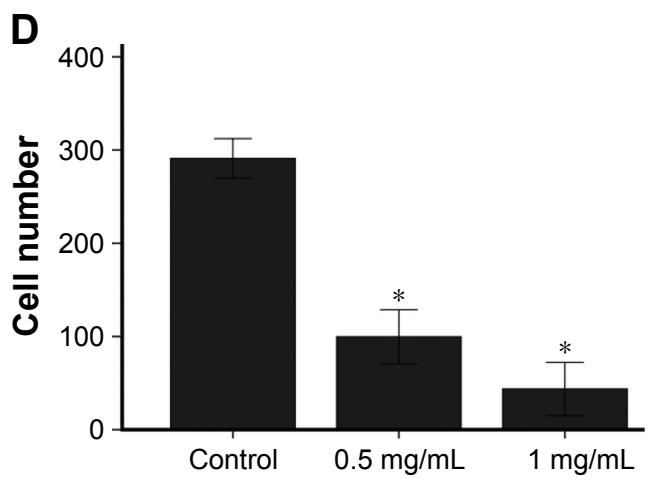

Figure 4 Ubenimex reduces the cell migration and invasion capacity of A375 cells.

Notes: Transwell migration and invasion assays were performed to determine the migration and invasion of the A375 cells. (A and B) The images above were obtained after culture for the indicated times (48 hour) in control medium or different doses of ubenimex. (C and D) Quantification was performed by counting the number of cells that passed through the small well using a high-power microscope. ${ }^{*} P<0.05$ vs the untreated control cells. Data are expressed as the mean \pm SD of three independent experiments.

Abbreviation: UB, ubenimex.

A375

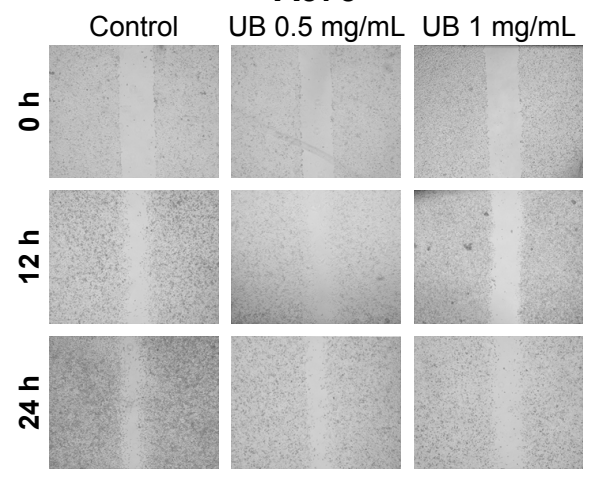

A375

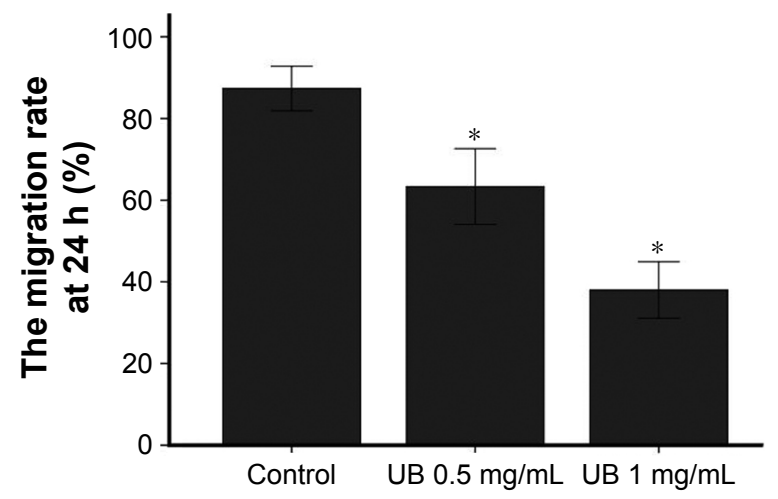

A2058

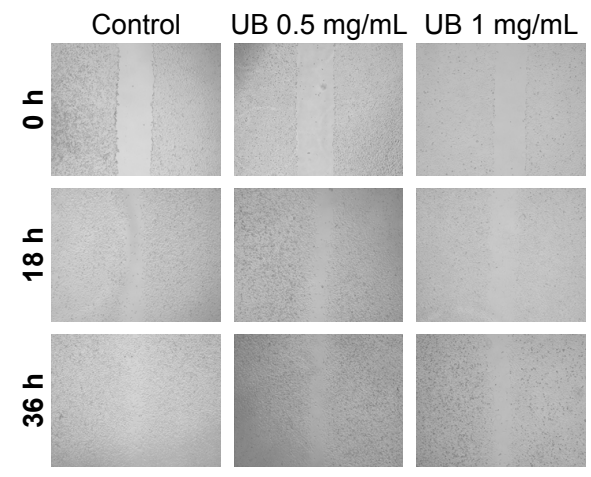

A2058

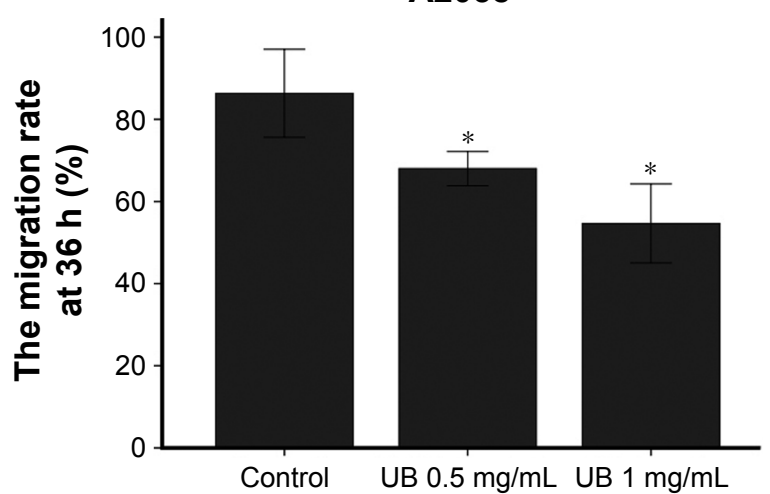

Figure 5 Ubenimex reduces the A375 and A2058 migration ability.

Notes: Wound healing migration assays were performed to determine the motility of the A375 and A2058 cells. Images of representative wells are shown after the indicated times of culture in control medium or medium with 0.5 or $1 \mathrm{mg} / \mathrm{mL}$ ubenimex. Quantification was performed by measuring the area migrated at different time points compared to the scratch area at $0 \mathrm{~h}$. Values represent the mean \pm SD of three independent experiments. $* P<0.05$ vs control untreated cells.

Abbreviation: UB, ubenimex. 

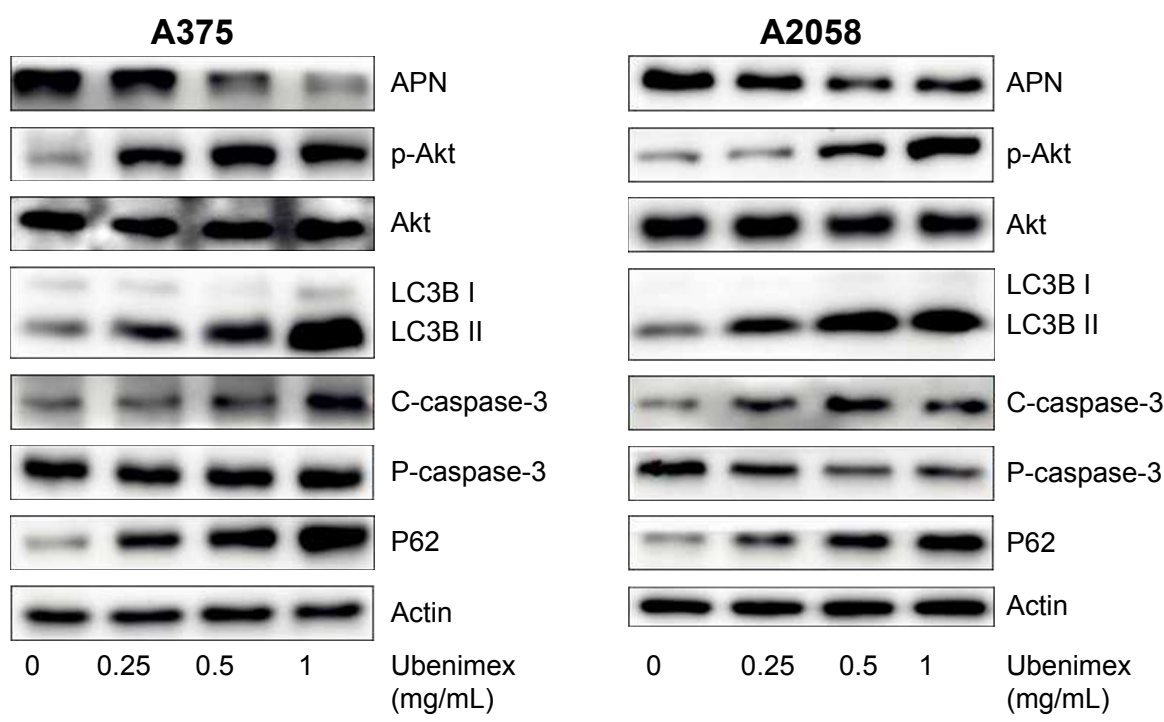

Figure 6 Ubenimex promotes the protein of autophagy and apopotosis in melanoma cells.

Notes: Western blotting of APN, p-Akt, Akt, LC3B, cleaved-caspase-3, pro-caspase-3, and P62 expression in A375 and A2058 cells after treatment for 48 h with ubenimex $(0.1,0.5$, or I mg/mL). Actin is shown as a loading control. Quantification was performed by densitometric analysis and normalized to actin levels.

Abbreviation: APN, Aminopeptidase N.
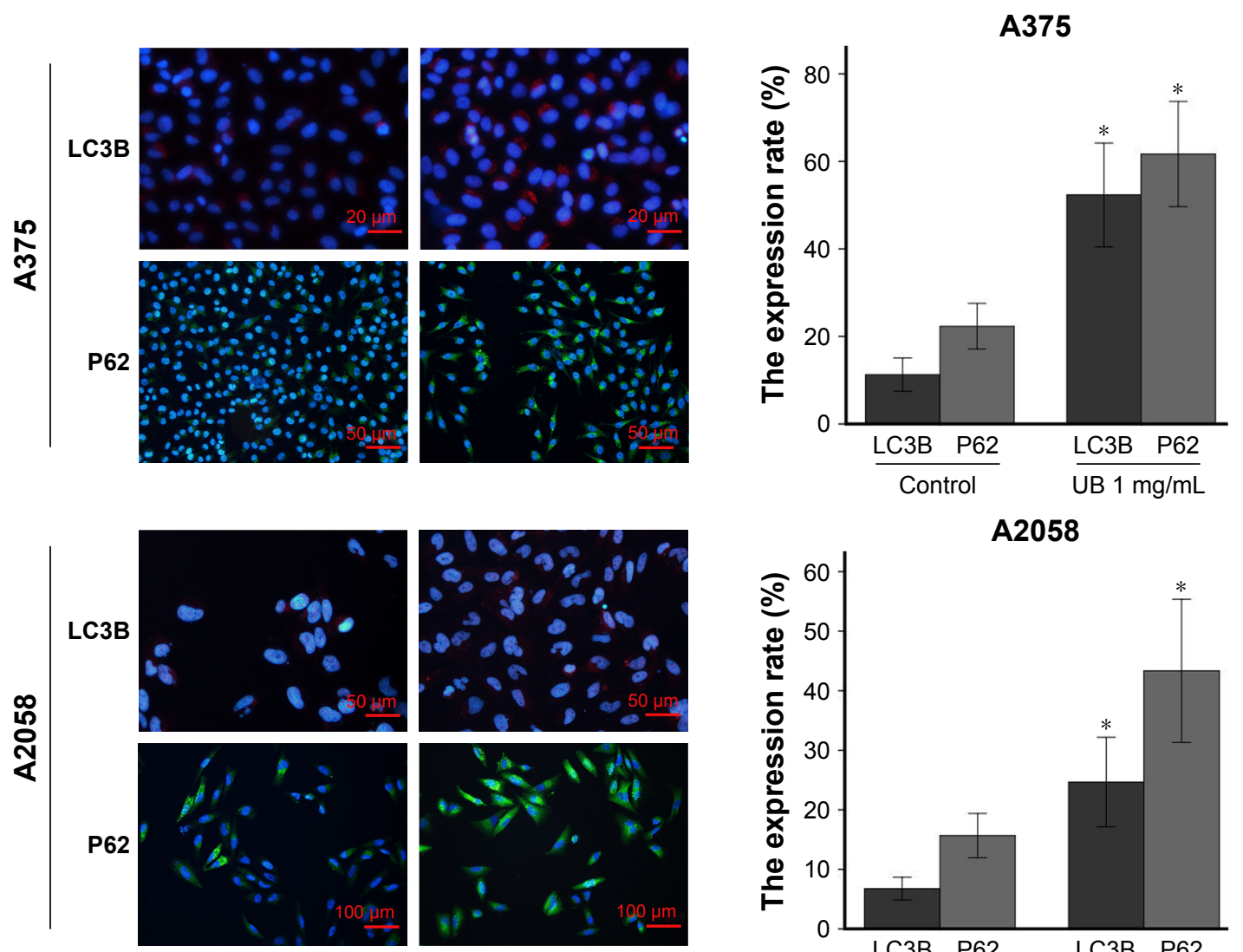

Control
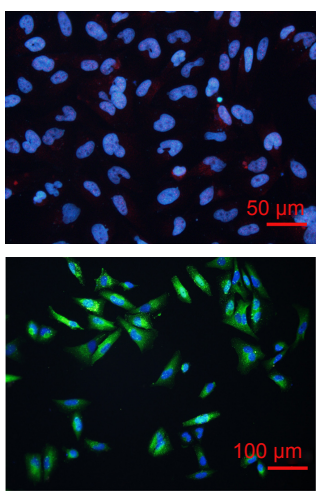

UB $1 \mathrm{mg} / \mathrm{mL}$

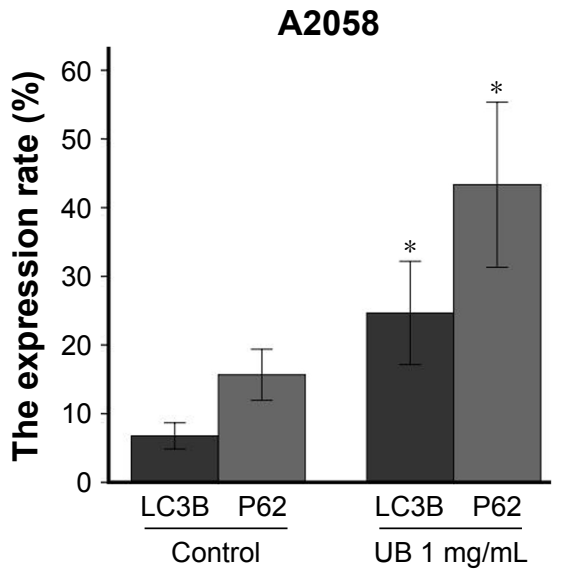

Figure 7 Ubenimex promotes the expression of LC3B and P62 in both A375 and A2058 cells.

Notes: Immunofluorescence studies were performed to assess LC3B and P62 expression levels. Immunofluorescence showed the expression of LC3B and P62 in A375 and A2058 cells after treatment for $48 \mathrm{~h}$ with ubenimex $(\mathrm{I} \mathrm{mg} / \mathrm{mL})$. Quantification was performed by evaluating the expression rate $(\%)$ under a high-power microscope. $* P<0.05$ vs the untreated control cells. Data are expressed as the mean \pm SD of three independent experiments.

Abbreviation: UB, ubenimex. 

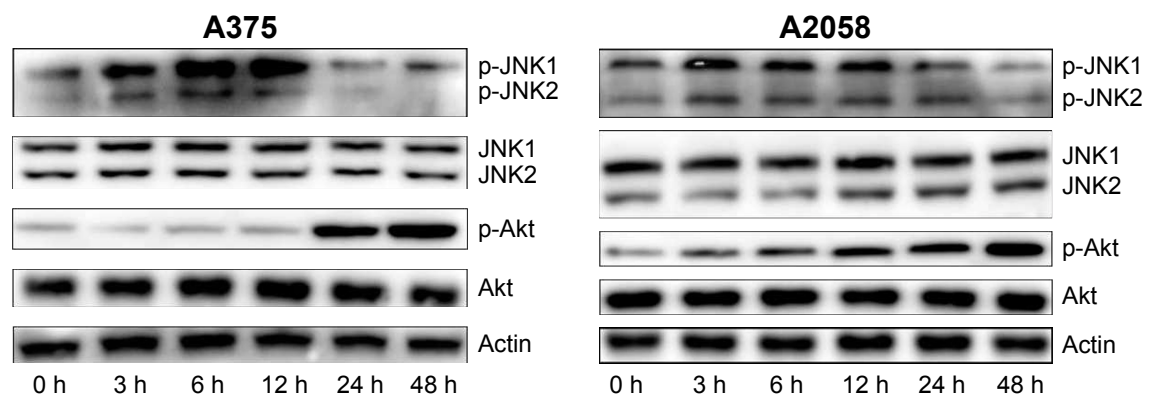

Figure 8 Ubenimex influences the expression of p-JNK and p-Akt in melanoma cells. Notes: Western blotting of JNK, p-JNK, Akt, and p-Akt expression in A375 and A2058 cells after treatment for different times with ubenimex (I mg/mL).

(PF-04691502, Selleck) to the ubenimex treatment. When combined with the Akt inhibitor, the effect of CCK-8 on cell proliferation was significantly inhibited, and cell death increased.

\section{Discussion}

Ubenimex continued to function as an APN inhibitor in A375 and A2058 cells. The inhibition of APN seems to be related to tumor metastasis, and reports have shown its function in many cancers, such as lung, pancreas, and colon cancers. ${ }^{17-19}$ In malignant melanoma cells, cell-surface APN expression could correlate to an invasive phenotype, and, through the inhibition of APN and ICAM, cell metastasis has been shown to significantly weaken. ${ }^{5}$ In particular, a previous study demonstrated that ubenimex, as an APN inhibitor, could inhibit tumor angiogenesis and may influence tumor growth and metastasis in B16-BL6 melanoma cells in vivo. ${ }^{20}$ However, there are few reports on the function of ubenimex in melanoma cells in vivo. Therefore, through Western blot analysis, when we added ubenimex, APN expression in A375 and A2058 cells was suppressed. These results are very consistent with previous findings in prostate cancer cells. ${ }^{15}$ Furthermore, matrigel invasion and migration assays were used to determine the migratory and invasion capacity of A375 cells. The capacity of A375 cells decreased with
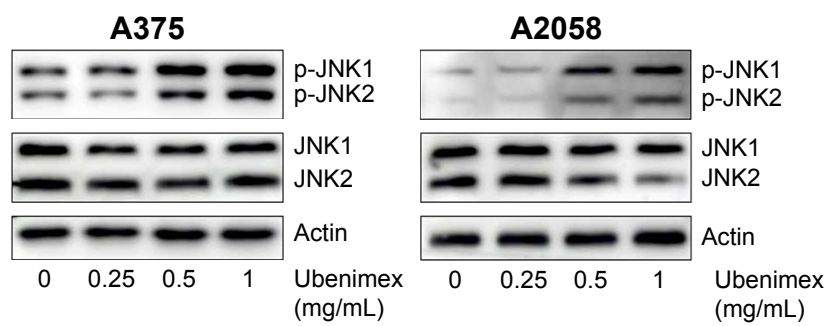

Figure 9 Ubenimex up-regulated the expression of JNK in melanoma cells. Note: Western blotting of JNK and p-JNK expression in A375 and A2058 cells after treatment for $6 \mathrm{~h}$ with ubenimex $(0.1,0.5$, or $1 \mathrm{mg} / \mathrm{mL})$. the use of ubenimex. Thus, we concluded that, in malignant melanoma cells, ubenimex could inhibit the ability to metastasize through the inhibition of APN.

Ubenimex could cause apoptosis and autophagic cell death in A375 and A2058 cells. Cell death is divided into programmed cell death and non-programmed death. Programmed cell death, which is often called apoptosis, is caspase dependent, whereas autophagic cell death is caspase independent. ${ }^{6}$ Both can cause tumor cell death. Although autophagy-associated cell death is different from apoptosis, there is sometimes a link between autophagy and apoptosis. ${ }^{21}$ In addition, the link is affected by cellular processes and determines whether the cells die or survive. In our previous study, ubenimex always served as an anti-tumor drug and induced cell death in prostate and renal cell carcinoma. ${ }^{14,15}$ Here, in malignant melanoma cells, we used 3MA to determine the presence of induced autophagic cell death. In addition, we found that ubenimex also induced caspase-related apoptosis, thus showing its anti-tumor capabilities. In our study, the apoptosis of malignant melanoma cells was assessed by Annexin V-PE/7AAD staining and Western blotting. Flow cytometry analysis showed that A375 and A2058 cell death increased with ubenimex treatment. Western blotting also demonstrated that caspase-3 was increased in this context. Thus, we detected a mixed phenotype of apoptosis and autophagic cell death in response to ubenimex treatment in malignant melanoma cells. Therefore, we concluded that ubenimex induced mixed programmed cell death in malignant melanoma cells.

Among mammals, there are three JNK genes: JNK1, JNK2, and JNK3. ${ }^{22}$ JNK1 and JNK2 are commonly expressed, while JNK3 is mainly manifested in the brain, heart, and testis. ${ }^{22}$ JNK, also known as stress-activated protein kinase, is closely related to apoptosis and autophagy. ${ }^{23-25}$ Moreover, the JNK signaling pathway is often stimulated by increased pressures 
from the external environment, including UV irradiation, oxidative stress, and drug treatment, which can induce apoptosis and autophagic cell death. ${ }^{23}$ Activation of the JNK pathway appears to lead to tumor cell death, including induced apoptosis and autophagy-related cell death. ${ }^{26}$ Here, we used Western blotting to analyze the JNK expression regulated by ubenimex. We found that JNK expression went up shortly before the drug was added, but, as time progressed, JNK expression decreased. We performed Western blotting at $6 \mathrm{~h}$ after treatment with ubenimex, and p-JNK expression was up-regulated. To determine the mechanism of cell death induced by the JNK pathway, we used a JNK inhibitor. In reference to the Western blot results, the JNK inhibitor was used to inhibit the increase in JNK in the first $6 \mathrm{~h}$. The inhibition of JNK decreased tumor cell death and weakened the inhibition of proliferation. Thus, we can conclude that the JNK pathway is involved in response to treatment with ubenimex.

The JNK pathway can induce not only caspase-related apoptosis, but also P62-related autophagy. ${ }^{27}$ P62 protein (sequestosome 1) is a selective substrate that plays a key role in forming cytoplasmic inclusion bodies. ${ }^{27}$ In particular, the stress tumor cells face induces the activation of JNK and the expression of P62, and then autophagic cell death occurs. ${ }^{28}$ In our experiment, the alteration of LC3B expression was the same as that of P62. Therefore, activation of JNK may induce P62-related autophagic cell death in A375 and A2058 cells.

Phosphoinositide 3-kinase (PI3K)/Akt is mainly involved in cell death, and is considered to deliver survival signals to protect cells from cell death. ${ }^{29}$ Thus, many researchers have demonstrated that the inhibition of Akt could be a good method for cancer treatment. ${ }^{30}$ In melanoma, inhibition of the Akt pathway also suggested an anti-tumor effect, and the activation of Akt seemed to have a protective effect. ${ }^{31,32}$ Additionally, previous studies have revealed that stress activates the Akt signal transduction pathway in tumor cells, resulting in a protective effect. ${ }^{13}$ Through our experiments, the stress of ubenimex treatment also led to the activation of the Akt signaling pathway in melanoma cells. Over time, the activation effect became increasingly obvious. After we used an Akt inhibitor, we found a very significant induction of death and the inhibition of proliferation in melanoma cells. Therefore, the application of ubenimex could lead to the activation of the protective Akt pathway, which can be reversed by its specific inhibitors.

\section{Conclusion}

The results of this study demonstrated that ubenimex could inhibit cell proliferation, migration, and invasion, and induce autophagic cell death and apoptosis in A375 and A2058 cells, and these efforts might be associated with the downregulation of APN and up-regulation of the JNK pathway. In addition, when treated with ubenimex, the melanoma cells up-regulated protective Akt activation, and the combined use of an Akt inhibitor with ubenimex provided a good effect for inducing tumor cell death. Therefore, this study suggests that ubenimex might be an excellent adjunctive therapy in the treatment of melanoma, with improved results when combined with an Akt inhibitor.

\section{Acknowledgments}

Thanks are given for the funding provided by the National Natural Science Foundation (81400575), the Science and Technology Development Plan Project of Shandong Province, China (2014GSF118144), and the Shandong Provincial Natural Science Foundation (ZR2017MH091).

\section{Disclosure}

The authors report no conflicts of interest in this work.

\section{References}

1. Savoia P, Fava P, Nardò T, Osella-Abate S, Quaglino P, Bernengo MG. Skin metastases of malignant melanoma: a clinical and prognostic survey. Melanoma Res. 2009;19(5):321-326.

2. Gladfelter P, Darwish NHE, Mousa SA. Current status and future direction in the management of malignant melanoma. Melanoma Res. 2017;27(5):403-410.

3. Nguyen DX, Bos PD, Massagué J. Metastasis: from dissemination to organ-specific colonization. Nat Rev Cancer. 2009;9(4):274-284.

4. Liu S, Xie F, Wang H, et al. Ubenimex inhibits cell proliferation, migration and invasion in renal cell carcinoma: The effect is autophagyassociated. Oncol Rep. 2015;33(3):1372-1380.

5. Laube F. Immunoluminescent detection of intercellular adhesion molecule- 1 and aminopeptidase $\mathrm{N}$ on human melanoma cells. Anticancer Res. 2007;27(4A):2047-2052.

6. Xu JW, Li CG, Huang XE, Li Y, Huo JG. Ubenimex capsule improves general performance and chemotherapy related toxicity in advanced gastric cancer cases. Asian Pac J Cancer Prev. 2011;12(4):985-987.

7. Riaz Ahmed KB, Kanduluru AK, Feng L, Fuchs PL, Huang P. Antitumor agent 25-epi Ritterostatin GN1N induces endoplasmic reticulum stress and autophagy mediated cell death in melanoma cells. Int J Oncol. 2017;50(5):1482-1490.

8. Huang XL, Zhang H, Yang XY, et al. Activation of a c-Jun N-terminal kinase-mediated autophagy pathway attenuates the anticancer activity of gemcitabine in human bladder cancer cells. Anticancer Drugs. 2017; 28(6):596-602.

9. Wang G, Zhang T, Sun W, et al. Arsenic sulfide induces apoptosis and autophagy through the activation of ROS/JNK and suppression of Akt/mTOR signaling pathways in osteosarcoma. Free Radic Biol Med. 2017;106:24-37.

10. Sun ZL, Dong JL, Wu J. Juglanin induces apoptosis and autophagy in human breast cancer progression via ROS/JNK promotion. Biomed Pharmacother. 2017;85:303-312.

11. He JD, Wang Z, Li SP, et al. Vitexin suppresses autophagy to induce apoptosis in hepatocellular carcinoma via activation of the JNK signaling pathway. Oncotarget. 2016;7(51):84520-84532.

12. Wang Y, Zhang G, Jin J, Degan S, Tameze Y, Zhang JY. MALT1 promotes melanoma progression through JNK/c-Jun signaling. Oncogenesis. 2017;6(7):e365. 
13. Wu J, Hu G, Dong Y, et al. Matrine induces Akt/mTOR signalling inhibition-mediated autophagy and apoptosis in acute myeloid leukaemia cells. J Cell Mol Med. 2017;21(6):1171-1181.

14. Liu S, Wang X, Lu J, et al. Ubenimex enhances the radiosensitivity of renal cell carcinoma cells by inducing autophagic cell death. Oncol Lett. 2016;12(5):3403-3410.

15. Wang X, Niu Z, Jia Y, et al. Ubenimex inhibits cell proliferation, migration and invasion by inhibiting the expression of APN and inducing autophagic cell death in prostate cancer cells. Oncol Rep. 2016; 35(4):2121-2130.

16. Han L, Zhao Q, Liang X, et al. Ubenimex enhances Brd4 inhibition by suppressing HEXIM1 autophagic degradation and suppressing the Akt pathway in glioma cells. Oncotarget. 2017;8(28):45643-45655.

17. Ikeda $N$, Nakajima $Y$, Tokuhara $T$, et al. Clinical significance of aminopeptidase N/CD13 expression in human pancreatic carcinoma. Clin Cancer Res. 2003;9(4):1503-1508.

18. Tokuhara $\mathrm{T}$, Hattori $\mathrm{N}$, Ishida $\mathrm{H}$, et al. Clinical significance of aminopeptidase $\mathrm{N}$ in non-small cell lung cancer. Clin Cancer Res. 2006;12(13): 3971-3978.

19. Hashida H, Takabayashi A, Kanai M, et al. Aminopeptidase N is involved in cell motility and angiogenesis: Its clinical significance in human colon cancer. Gastroenterology. 2002;122(2):376-386.

20. Aozuka Y, Koizumi K, Saitoh Y, Ueda Y, Sakurai H, Saiki I. Antitumor angiogenesis effect of aminopeptidase inhibitor bestatin against B16-BL6 melanoma cells orthotopically implanted into syngeneic mice. Cancer Lett. 2004;216(1):35-42.

21. Xiang XY, Yang XC, Su J, et al. Inhibition of autophagic flux by ROS promotes apoptosis during DTT-induced ER/oxidative stress in HeLa cells. Oncol Rep. 2016;35(6):3471-3479.

22. Kops GJ, Dansen TB, Polderman PE, et al. Forkhead transcription factor FOXO3a protects quiescent cells from oxidative stress. Nature. 2002;419(6904);316-321.
23. Deng R, Li W, Guan, Z et al. Acetylcholinesterase expression mediated by c-Jun-NH2-terminal kinase pathway during anticancer drug-induced apoptosis. Oncogene. 2006;25(53):7070-7077.

24. Mizukami Y, Yoshioka K, Morimoto S, Yoshida Ki. A novel mechanism of JNK1 activation. Nuclear translocation and activation of JNK1 during ischemia and reperfusion. J Biol Chem. 1997;272(26): 16657-16662.

25. Zhang P, Miller BS, Rosenzweig SA, Bhat NR. Activation of C-jun $\mathrm{N}$-terminal kinase/stress-activated protein kinase in primary glial cultures. J Neurosci Res. 1996;46(1):114-121.

26. He JD, Wang Z, Li SP, et al. Vitexin suppresses autophagy to induce apoptosis in hepatocellular carcinoma via activation of the JNK signaling pathway. Oncotarget. 2016;7(51):84520-84532.

27. De Luca A, Carpanese D, Rapanotti MC, et al. The nitrobenzoxadiazole derivative MC3181 blocks melanoma invasion and metastasis. Oncotarget. 2017;8(9):15520-15538.

28. Go DH, Lee YG, Lee DH, et al. 3-Decylcatechol induces autophagymediated cell death through the IRE $1 \alpha / \mathrm{JNK} / \mathrm{p} 62$ in hepatocellular carcinoma cells. Oncotarget. 2017;8(35):58790-58800.

29. Krajarng A, Chulasiri M, Watanapokasin R. Etlingera elatior Extract promotes cell death in B16 melanoma cells via down-regulation of ERK and Akt signaling pathways. BMC Complement Altern Med. 2017;17(1):415.

30. Díaz M, González R, Plano D, Palop JA, Sanmartín C, Encío I. A diphenyldiselenide derivative induces autophagy via JNK in HTB-54 lung cancer cells. J Cell Mol Med. 2018;22(1):289-301.

31. Pappalardo F, Russo G, Candido S, et al. Computational modeling of PI3K/AKT and MAPK signaling pathways in melanoma cancer. PLoS One. 2016;11(3):e0152104.

32. Tang R, Xu X, Yang W, et al. MED27 promotes melanoma growth by targeting AKT/MAPK and NF- $\mathrm{KB} / \mathrm{iNOS}$ signaling pathways. Cancer Lett. 2016;373(1):77-87.
OncoTargets and Therapy

\section{Publish your work in this journal}

OncoTargets and Therapy is an international, peer-reviewed, open access journal focusing on the pathological basis of all cancers, potential targets for therapy and treatment protocols employed to improve the management of cancer patients. The journal also focuses on the impact of management programs and new therapeutic agents and protocols on

\section{Dovepress}

patient perspectives such as quality of life, adherence and satisfaction. The manuscript management system is completely online and includes a very quick and fair peer-review system, which is all easy to use. Visit http://www.dovepress.com/testimonials.php to read real quotes from published authors. 